\title{
Short-Chain Fatty Acids Decrease the Frequency of Spontaneous Contractions of Longitudinal Muscle via Enteric Nerves in Rat Distal Colon
}

\author{
Shigeyuki ONO*†, Shin-ichiro KARAKI*, and Atsukazu KUWAHARA* \\ *Laboratory of Physiology, Graduate School of Nutritional and Environmental Sciences, Institute for Environmental \\ Sciences, University of Shizuoka, 52-1, Yada, Shizuoka, 422-8526 Japan; and tKao Corporation, 1-3, \\ Bunka 2-choume, Sumida-ku, Tokyo, 131-8501 Japan
}

\begin{abstract}
Short-chain fatty acids (SCFAs) produced by the bacterial fermentation of carbohydrates in the cecum and proximal colon are reported to modify colonic motility as a luminal factor. Besides the physical stimuli in the distal colon, SCFAs in the intestinal lumen also seem to affect colonic motility under physiological concentrations. This study therefore used fasted rats to investigate the effect of SCFAs on the spontaneous contractions of longitudinal muscle (LM) in rat distal colon, including mucosa in vitro. The frequency of spontaneous contractions of LM strips from the distal colon was 9.4 \pm 0.5 contractions $/ 20 \mathrm{~min}$. The exogenous addition of $>5 \mathrm{~mm}$ SCFAs decreased the frequency of spontaneous contractions of the LM to $6.1 \pm$
\end{abstract}

0.8 contractions $/ 20 \mathrm{~min}$. Among SCFAs, only acetate elicited this inhibitory response. TTX and the combination of hexamethonium and granisetron abolished SCFA-induced inhibitory response, suggesting that this inhibitory response is mediated via the ENS, including nicotinic and $5-\mathrm{HT}_{3}$ receptors. In conclusion, it is suggested that SCFAs in rat distal colon decrease the frequency of spontaneous contractions of the LM and that SCFAs may contribute to colonic motility, including the peristaltic reflex, by regulating the frequency of spontaneous contractions of the LM through the enteric nervous system (ENS). [The Japanese Journal of Physiology 54: 483-493, 2004]

Key words: SCFA; longitudinal muscle, frequency, rat colon, enteric nervous system.

$\mathrm{O}_{\mathrm{n}}$ ne of the most important events in the large intestinal lumen is the production of short-chain fatty acids (SCFAs) through the bacterial fermentation of carbohydrates in the cecum and proximal colon of monogastric mammals. SCFAs are the major anions of colonic contents, including mainly acetate, propionate, and butyrate in a molar ratio of 57:21:22 in the human right colon, respectively. The total concentration is usually $\sim 130 \mathrm{mM}$ [1]. The effects of SCFAs on gastrointestinal motility have been extensively investigated in previous works [2-6]. However, the effects of SCFAs on colonic motility are controversial. For example, Yajima [7] has reported that a low concentration $(0.1 \mathrm{mM})$ of propionate and butyrate, but not acetate, causes a transient contrac- tion in isolated segments and strips of rat colon via enteric reflex. It has also been reported that a high concentration $(500 \mathrm{mM})$ of SCFAs infused into rat proximal colon reduces colonic motility and increases transit rate; SCFAs decrease the ratio of orally to aborally propagated activity to accelerate colonic transit, and this effect may be mediated through a neuroendocrine mechanism involving peptide YY (PYY) [8]. Fukumoto et al. [9] have recently reported that the administration of SCFAs (100 mM) into the proximal colon significantly increased colonic motility via vagovagal reflex involving the release of 5-HT from enterochromaffin cells (EC cells) in rats; the highamplitude contractions induced by SCFAs in the proximal colon migrated to the middle and distal colon,

Received on July 28, 2004; accepted on October 25, 2004

Correspondence should be addressed to: A. Kuwahara, Laboratory of Physiology, Institute for Environmental Sciences, University of Shizuoka, 52-1 Yada, Shizuoka, 422-8526 Japan, Tel/Fax: +81-54-264-5707, E-mail: kuwahara@sea.u-shizuoka-ken.ac.jp 
suggesting that SCFAs promote propulsive movement.

It is well known that the peristaltic reflex in the distal colon occurs through the physical stimuli of fecal bulk [10]. Fecal pellets are made in the middle and distal colon, and the activity of fermentation in these regions is weak or virtually absent [11]. However, the concentration of SCFAs contained in fecal pellets is still about $100 \mathrm{mM}$ [1]. This suggests that SCFAs migrate with fecal pellets in the middle and distal colon. Therefore we considered that not only fecal bulk, but also SCFAs contained in fecal pellets might influence colonic motility as a chemical stimulus. However, the effects of SCFAs on the motor activity, especially on the frequency of spontaneous contractions in rat distal colon, are still unclear.

It has been reported that longitudinal (LM) as well as circular muscle (CM) layers are also important during peristalsis because they exhibit synchronous movements in guinea pig distal colon; the LM and CM synchronously contracted oral (ascending excitation) to, and relaxed anal (descending inhibition) to, a peristaltic wave [10]. As for the role of the LM during peristalsis, it has been suggested that oral contraction of the LM tends to pull the intestine over the rear of a pellet, the simultaneous contraction of both muscle layers ensuring that contraction of the LM also contributes to propulsion by providing a vector of force along the intestine [10] When recorded mechanically, in vitro and in vivo colonic motor activity in most species, including mice and rats, is characterized by two distinct types of contractions: (1) rhythmic phasic contractions, and (2) spontaneous contractions, which are also termed giant contractions (GCs) by Gonzalez and Sarna [12]. It is thought that the rhythmic phasic contractions are regulated by slow waves superimposed with spikes because their maximum frequency is the same as that of slow waves $[13,14]$. It has been recently reported that spontaneous contractions in longitudinal as well as circular colonic muscle strips of mice are of similar form, frequency, and pharmacology to circular muscle colonic migrating motor complexes (CMMCs) [15], which are also termed giant migrating contractions (GMCs) by Gonzalez and Sarna [12, 13]. Because the mouse CMMCs resemble peristaltic waves in their frequency and time course, and because they migrate over half the length of the colon, CMMCs are thought to be peristaltic in nature [16]. Furthermore, GMCs are large-amplitude and long-duration contractions that migrate to other parts of the colon and are associated with mass movements and defecation [17-19]. It is reasonable to speculate that spon- taneous contractions of the LM are appropriate for considering the regulation of colonic motor activities, including peristalsis and defecation. Therefore in the present study we investigated the effects of SCFAs on in vitro motor activity of rat distal colon using spontaneous contractions of the LM with mucosa as an index.

\section{METHODS}

Tissue preparation. Male Wistar rats (200-350 g, Charles River Japan Inc., Yokohama, Japan) were allowed food and water ad libitum and were usually fasted about $24 \mathrm{~h}$ before experiments. The animals were anaesthetized with diethyl ether and then killed by decapitation according to the method approved by the Guide for Animal Experimentation of National Institute for Physiological Sciences of Japan. Distal colon, the segment between the entrances of the left branches of the middle colonic artery and the upper side of the rectum, was removed and placed in KrebsRinger solution kept on ice. The solution contained (in $\mathrm{mM}$ ) $117 \mathrm{NaCl}, 4.7 \mathrm{KCl}, 1.2 \mathrm{MgCl}_{2}, 1.2 \mathrm{NaH}_{2} \mathrm{PO}_{4}$, $25 \mathrm{NaHCO}_{3}, 2.5 \mathrm{CaCl}_{2}$, and 11 glucose and was bubbled with a gas mixture of $95 \% \quad \mathrm{O}_{2}$ and $5 \% \quad \mathrm{CO}_{2}$. Segments of distal colon were opened along the mesenteric border, cleaned, and pinned flat on a petri dish with a silicon rubber base. Full-thickness strips ( $2 \mathrm{~mm}$ wide and $1-1.5 \mathrm{~cm}$ long) were cut along the longitudinal axis and mounted in a $15 \mathrm{ml}$ organ bath filled with Krebs-Ringer solution at $37^{\circ} \mathrm{C}$, which was continuously aerated with a mixture of $95 \% \mathrm{O}_{2}$ and $5 \% \mathrm{CO}_{2}$.

\section{Measurement of spontaneous contrac-}

tions. The strips were placed under a resting tension of $0.5 \mathrm{~g}$, and changes in length were recorded with isotonic transducers (NEC San-ei Instrument, Ltd., Tokyo, Japan). Output was monitored by MacLab (AD Instruments, Cattle Hill, Australia). The strips were left to equilibrate for at least $120 \mathrm{~min}$. A count of the number of spontaneous contractions in $20 \mathrm{~min}$ indicated the frequency of spontaneous contractions.

A representative trace of the spontaneous contractions recorded mechanically from the LM strips of rat distal colon is shown in Fig. 2A. It is known that spontaneous contractions (or GCs) corresponding to GMCs are large-amplitude and long-duration contractions [12]. The amplitude of the contractions was about half that of a contraction induced by $\mathrm{ACh}$ $\left(10^{-6} \mathrm{M}\right)$ at the end of the experiment. Therefore these contractions were confirmed to be spontaneous and ones we will regard as an index in the present study. It has been reported that small-amplitude and short- 
duration contractions appearing between spontaneous contractions are rhythmic phasic contractions regulated by slow waves superimposed with spikes $[13,14]$.

Experimental protocols. The effect of SCFAs on the frequency of spontaneous contractions of the LM was determined by the following protocol: After the equilibration of the strips, the frequency of spontaneous contractions was measured for $20 \mathrm{~min}$ (control), various concentrations of SCFAs (0 and 0.1-10 $\mathrm{mM}$ ) were then added, and after a further $30 \mathrm{~min}$ equilibration, the frequency of spontaneous contractions was measured again for $20 \mathrm{~min}$ (measurement). The SCFAs used were a mixture of sodium acetate, sodium propionate, and sodium butyrate in a molar ratio of 75:35:20, respectively, based on SCFA contents in rat cecum [20]. To ensure that these effects would not be from an increase in the concentration of sodium ion or in osmotic pressure caused by SCFAs, 10 $\mathrm{mM} \mathrm{NaCl}$ was added to the bathing solution, and the spontaneous contractions were measured.

To investigate the involvement of the ENS in the effect of SCFAs on the spontaneous contractions, neural blockades or antagonists were added before SCFAs were added. The respective control was measured for $20 \mathrm{~min}$ before the addition of these drugs. When there were no apparent alterations in the frequency of spontaneous contractions of the LM because of the addition of drugs themselves (tetrodotoxin $\left[\mathrm{TTX}, 10^{-6} \mathrm{M}\right]$, hexamethonium $[\mathrm{C} 6$, $\left.10^{-4} \mathrm{M}\right]$, granisetron $\left[10^{-6} \mathrm{M}\right]$, and the combination of C6 and granisetron), SCFAs (5 mM) were added 10 (TTX, C6) or 20 min (granisetron, combination) after the drugs were. Since the addition of $N^{\omega}$-nitroL-arginine (L-NNA, $10^{-4} \mathrm{M}$ ) apparently altered the frequency of spontaneous contractions of the LM, the frequency of spontaneous contractions 10 min after the addition of this drug was measured for $20 \mathrm{~min}$ (additional control); then the SCFAs $(5 \mathrm{mM}$ ) were added.

The effects of individual SCFAs (acetate, propionate, and butyrate) were also tested. Moreover, after the measurement of frequency in the presence of acetate $(5 \mathrm{mM})$ (measurement), propionate $(2 \mathrm{mM})$ was further added, and $30 \mathrm{~min}$ after the addition of propionate, the frequency was measured for $20 \mathrm{~min}$ (additional measurement).

In some experiments, fed rats were used to explore the possibility that SCFAs contained in fecal pellets might influence the frequency of spontaneous contractions of the LM. To test the influence of SCFAs on the frequency of spontaneous contractions of the LM in fed rats, SCFAs ( $5 \mathrm{mM}$ ) were also added after the measurement of control.
Drugs. Sodium acetate, sodium propionate, and sodium butyrate were purchased from Wako Pure Chemical Industries, Ltd. (Osaka, Japan), tetrodotoxin, hexamethonium bromide, acetylcholine chloride, and $N^{\omega}$-nitro-L-arginine from Sigma Chemical Co. (St. Louis, MO, USA). Granisetron was a gift from Fujisawa Pharmaceutical Co., Ltd. (Osaka, Japan). All drugs were dissolved in distilled water. The solutions containing drugs in a volume within $150 \mu \mathrm{l}$ were added to the bathing solution.

Statistics. The data are expressed as means \pm SEM. The differences in the data were evaluated by Student's $t$-test or ANOVA, followed by a Dunnett test. The $\mathrm{P}$ values of less than 0.05 were taken as a statistically significant difference.

\section{RESULTS}

\section{Effect of exogenous SCFAs on the fre- quency of spontaneous contractions of the}

LM. The frequency of spontaneous contractions of the LM was $9.4 \pm 0.5$ contractions $/ 20 \mathrm{~min}(n=7)$. To evaluate the effect of SCFAs on the frequency of spontaneous contractions of the LM in rat distal colon, various concentrations ( 0 and $0.1-10 \mathrm{mM}$ ) of SCFAs were added to the bathing solution, and changes in the frequency, amplitude and duration of spontaneous contractions of the LM were studied. SCFAs gradually decreased the frequency of spontaneous contractions of the LM, and about 20 min after the addition of SCFAs, the frequency of spontaneous contractions had stabilized (Fig. 1A). There was no significant difference in the frequency of spontaneous contractions of the LM among respective controls ( $n$ $=6-8, P>0.05$ by ANOVA, Fig. 1B). At more than $1 \mathrm{mM}$ of SCFAs, they concentration-dependently decreased the frequency of spontaneous contractions of the LM and reached a maximum at $5 \mathrm{mM}(6.1 \pm$ 0.8 contractions $/ 20$ min, $n=8, P<0.05$ by Student's $t$-test, Fig. 1B). The representative trace of $5 \mathrm{mM}$ SCFAs inhibitory effect on the frequency of spontaneous contractions of the LM is shown in Fig. 1A. However, the SCFAs did not affect the amplitude or duration of spontaneous contraction of the LM (Fig. 1A). The ratio of average amplitude of spontaneous contractions of the LM in "measurement" to that in "control" was $103.6 \pm 4.6 \%$ (5 mM, $n=8)$. Similarly, the ratio of average duration of spontaneous contractions of the LM in "measurement" to that in "control" was $100.2 \pm 6.3 \%$ ( $5 \mathrm{~mm}, n=8)$.

Sometimes the SCFAs evoked a transient contraction, but the transient contraction did not affect 

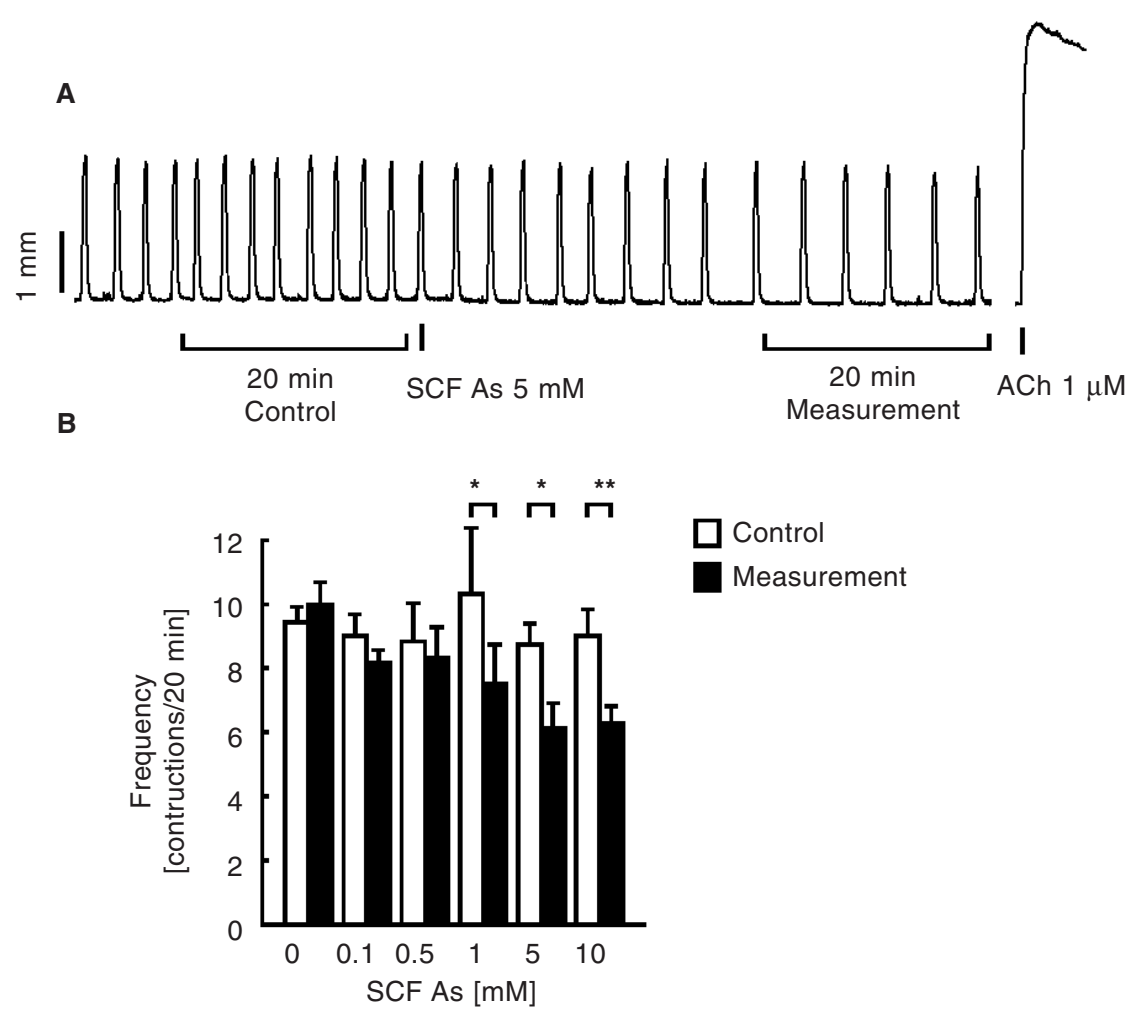

Fig. 1. Inhibitory effect of SCFAs on the frequency of spontaneous contractions of the LM. A, representative trace of $5 \mathrm{mM}$ SCFA-induced inhibitory response. B, there were no significant differences in the frequency of spontaneous contractions among respective controls when differences in the data were evaluated by ANOVA. The effect of SCFAs $(0-10 \mathrm{mM})$ on the frequency of spontaneous contractions of the LM (measurement) was significant at more than $1 \mathrm{mM}$ SCFAs to each control, when differences in the data were evaluated by Student's $t$-test. This effect of SCFAs on the frequency of spontaneous contractions of the LM reached a maximum at $5 \mathrm{mM}$. open squares: control. Solid squares: measurement. The values are means \pm SEM, $n=6-8 .{ }^{*} P<0.05,{ }^{\star *} P<0.01$, significantly different from control.

the frequency of spontaneous contractions of the LM that was reduced by exogenous SCFAs.

Since the effect of SCFAs on the frequency of spontaneous contractions of the LM reached a maximum at $5 \mathrm{mM}$, in the following experiments a submaximal concentration of SCFAs of $5 \mathrm{mM}$ was used to investigate the effect of SCFAs on the frequency of spontaneous contractions of the LM in rat distal colon.

Influence of sodium ion and high osmolarity on the frequency of spontaneous contractions of the LM. Because $\mathrm{pH}$ in the colon is neutral ( $\mathrm{pH} \mathrm{6-7)}$ under normal conditions [21], SCFAs were used as sodium salts. Sodium salts of SCFAs have almost no effect on $\mathrm{pH}$ in an organ bath because they are nearly neutral. Therefore this indicates that the effect of SCFAs is not an influence of the change in $\mathrm{pH}$ of the bathing solution. However, it is possible that the effect of SCFAs on the frequency of spontaneous contractions of the LM was due to the effects of sodium ion $\left(\mathrm{Na}^{+}\right)$and/or high osmolarity. Thus to confirm that increases in the concentration of $\mathrm{Na}^{+}$and in osmolarity caused by SCFAs had no effect, the change in the frequency of spontaneous contractions of the LM was measured by using 10 $\mathrm{mM} \mathrm{NaCl}$ instead of SCFAs in the distal colon.

The results showed that $10 \mathrm{mM} \mathrm{NaCl}$ did not affect the frequency of spontaneous contractions of the LM (control: $9.3 \pm 1.6$ contractions/20 min; $10 \mathrm{mM} \mathrm{NaCl}$ : $8.7 \pm 1.2$ contractions $/ 20 \mathrm{~min}, n=6$ ). Thus the data suggest that the effect of SCFAs on the frequency of spontaneous contractions of the LM is due to the effect of SCFAs themselves.

Effects of individual SCFAs on the frequency of spontaneous contractions of the

LM. In the present study, the SCFAs used were a mixture of acetate, propionate, and butyrate based on the previous report [20]. The effects of individual SCFAs on the frequency of spontaneous contractions of the LM were tested.

Acetate $(5 \mathrm{mM})$ significantly decreased the frequency of spontaneous contractions of the LM from $8.8 \pm 0.3$ contractions $/ 20 \mathrm{~min}$ (control) to $5.8 \pm 0.3$ contractions/20 min (measurement) without a transient contraction immediately after the addition of acetate $(n=6, P<0.01$, Fig. $2, \mathrm{~A}$ and D). On the 
other hand, propionate $(5 \mathrm{mM})$ significantly increased the frequency of spontaneous contractions of the LM from $10.0 \pm 0.6$ contractions $/ 20 \mathrm{~min}$ (control) to 14.2 \pm 1.2 contractions $/ 20 \mathrm{~min}$ (measurement) with a transient contraction immediately after the addition of propionate ( $n=5, P<0.05$, Fig. 2 , B and E). Butyrate $(5 \mathrm{mM})$ induced a transient contraction, but did not affect the frequency of spontaneous contractions of the $\operatorname{LM}(n=6$, Fig. $2, \mathrm{C}$ and F).
Effect of propionate on acetate-induced inhibitory response. Only acetate elicited the inhibitory response decreasing the frequency of spontaneous contractions of the LM. On the other hand, propionate increased the frequency of spontaneous contractions of the LM. Since propionate coexists with acetate in SCFAs, the effect of propionate, in the presence of acetate, on the frequency of spontaneous contractions of the LM was examined.

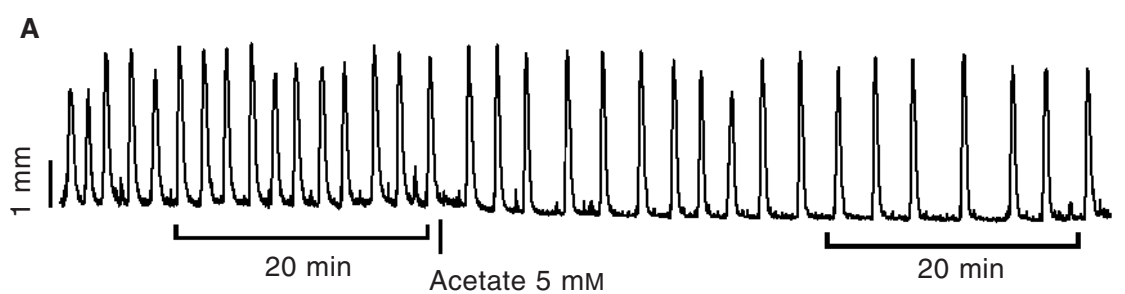

B

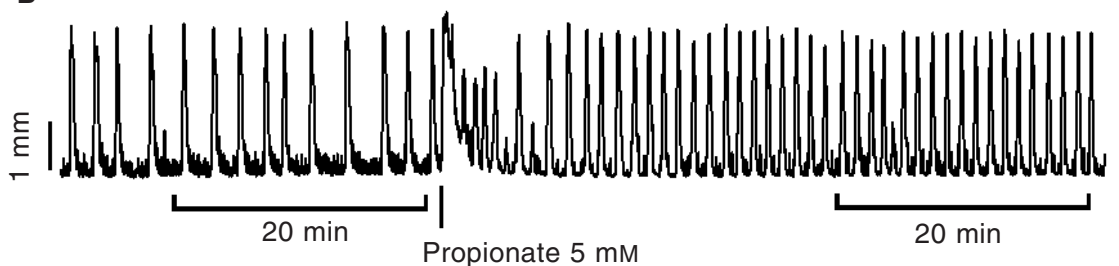

C
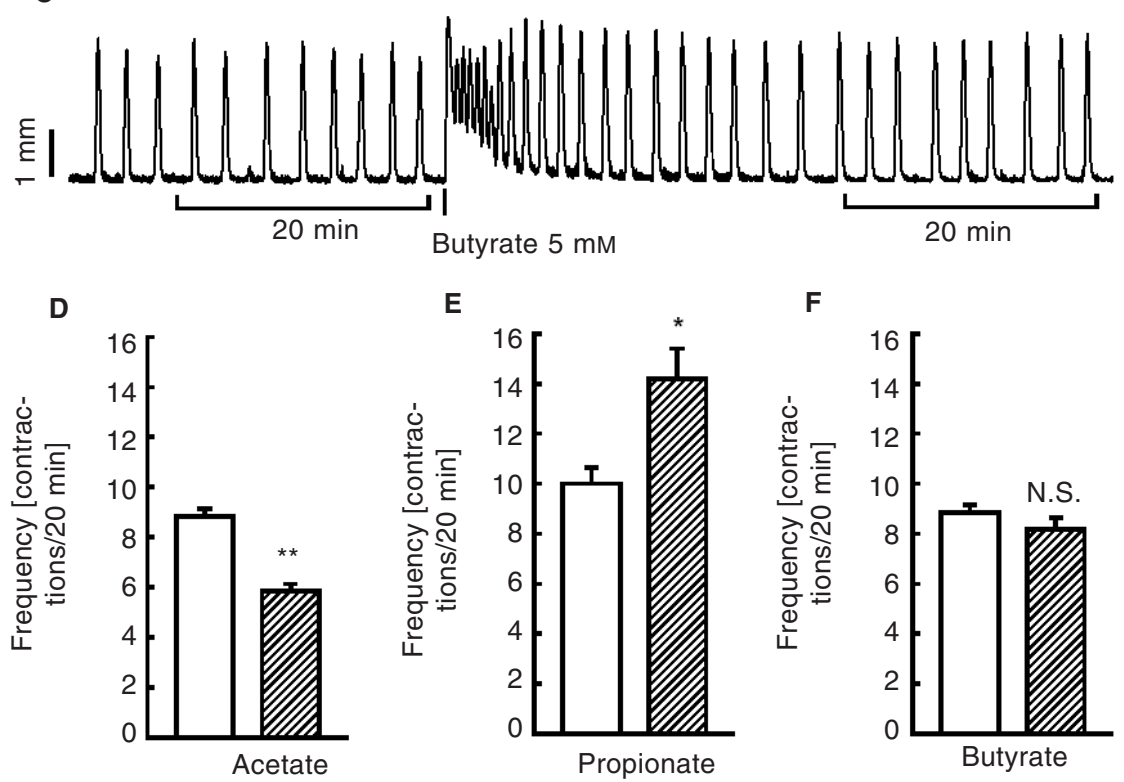

E

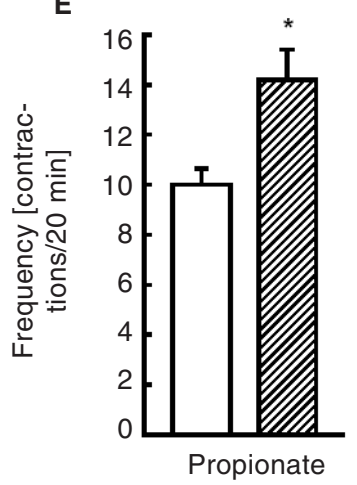

$\mathbf{F}$

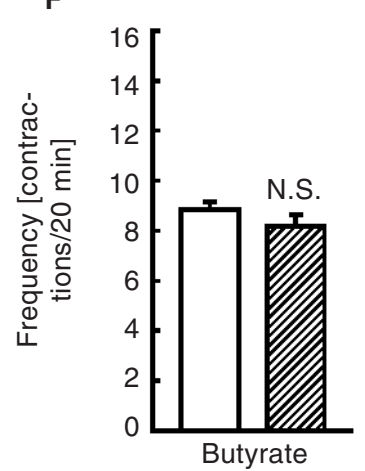

Fig. 2. Effects of individual SCFAs on the frequency of spontaneous contractions of the LM. A, representative trace showing that acetate $(5 \mathrm{mM})$ decreased the frequency of spontaneous contractions of the LM without a transient contraction immediately after its addition. B, representative trace showing that propionate (5 mM) increased the frequency of spontaneous contractions of the LM with a transient contraction immediately after its addition. C, representative trace showing that butyrate $(5 \mathrm{mM})$ caused a transient contraction. D, acetate $(5 \mathrm{~mm})$ significantly decreased the frequency of spontaneous contractions of the LM. The values are means \pm SEM, $n=6$. ${ }^{\star \star} P<0.01$, significantly different from control. E, propionate $(5 \mathrm{mM})$ significantly increased the frequency of spontaneous contractions of the LM. The values are means \pm SEM, $n=5$. ${ }^{\star} P<0.05$, significantly different from control. $\mathbf{F}$, butyrate $(5 \mathrm{mM})$ did not influence the frequency of spontaneous contractions of the LM. The values are means \pm SEM, $n=6$. 
Acetate $(5 \mathrm{mM})$ significantly decreased the frequency of spontaneous contractions of the LM from $9.3 \pm 0.3$ contractions $/ 20 \mathrm{~min}$ (control) to $6.0 \pm 0.4$ contractions $/ 20 \mathrm{~min}$ (measurement) $(n=4, P<0.01$, Fig. 3, A and B). After that, the addition of $2 \mathrm{~mm}$ propionate, which was used to mimic the molar ratio of propionate to acetate in SCFAs, neither affected the frequency of spontaneous contractions nor induced a transient contraction in the presence of acetate (additional measurement) ( $n=4$, Fig. 3, A and B).

Effect of tetrodotoxin on SCFA-induced inhibitory response. It has been known that the ENS influences intestinal motor activity [10]. Therefore, whether SCFA-induced inhibitory response in which exogenous SCFAs decreased the frequency of spontaneous contractions of the LM was mediated by the intrinsic nervous system in the intestine (the ENS) was tested by the use of tetrodotoxin (TTX).

TTX $\left(10^{-6} \mathrm{M}\right)$ itself did not affect the frequency of spontaneous contractions of the LM. However, TTX significantly prevented the SCFA-induced inhibitory response ( $n=6$, Fig. 4$)$. This result suggests that the SCFA-induced inhibitory response is due to TTXsensitive mechanisms.
Effects of some neural blockades on SCFAinduced inhibitory response. The result in Fig. 4 suggested that SCFA-induced inhibitory response is mediated by the ENS. Therefore some neural blockades other than TTX were used for further investigation.

Effect of an NOS-inhibitor on SCFA-induced inhibitory response. Nitric oxide (NO) as an inhibitory transmitter is known to influence the frequency of spontaneous contractions in rat colon [12]. For that reason, SCFA-induced inhibitory response might also be mediated by NO synthesis. Whether NO participates in SCFA-induced inhibitory response was tested by using L-NNA, a nitric oxide synthase inhibitor. L-NNA $\left(10^{-4} \mathrm{M}\right)$ significantly increased the frequency of spontaneous contractions of the LM from $9.6 \pm 0.5$ contractions $/ 20 \mathrm{~min}$ (control) to 13.0 \pm 0.5 contractions $/ 20$ min (additional control) $(n=$ $5, P<0.01$, Fig. 5). This result suggests that $\mathrm{NO}$ is always produced in rat distal colon, and inhibits spontaneous contractions to decrease their frequency under basal conditions. In the presence of L-NNA, the further addition of SCFAs significantly decreased the frequency of spontaneous contractions to $11.0 \pm 0.7$

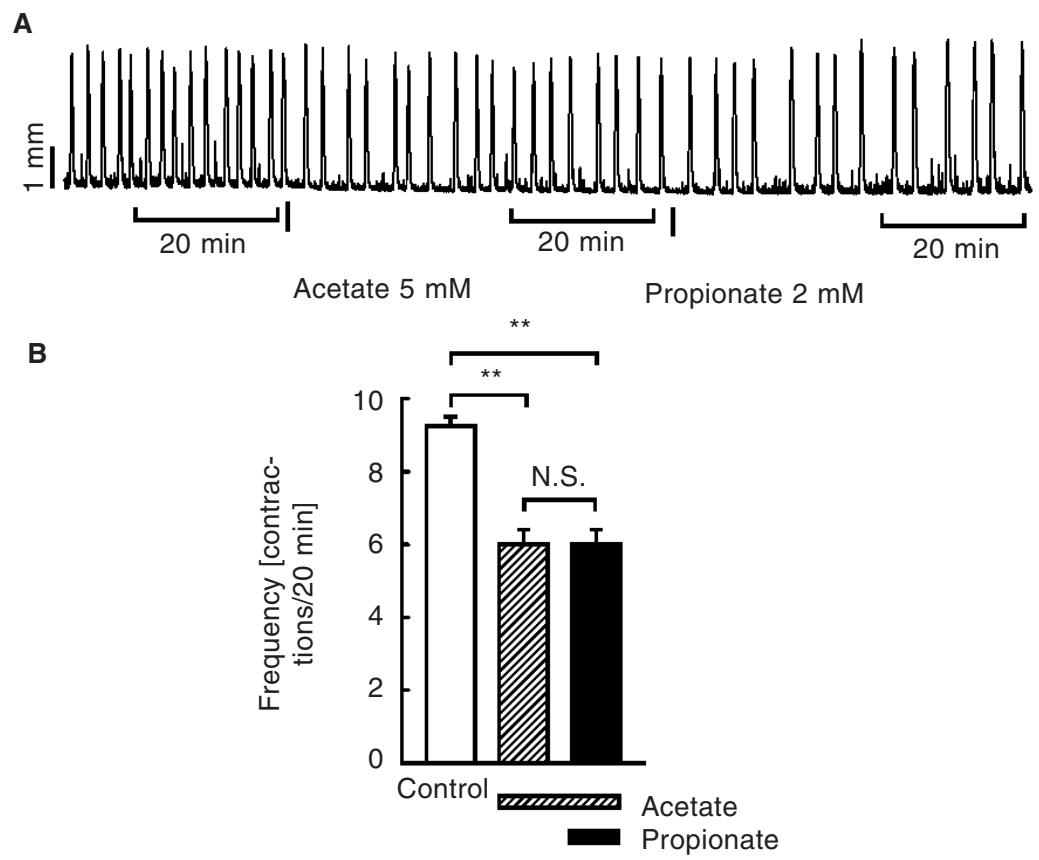

Fig. 3. Effect of propionate on acetate-induced inhibitory response. A, representative trace showing that the further addition of $2 \mathrm{mM}$ propionate neither affected the frequency of spontaneous contractions of the LM reduced by $5 \mathrm{mM}$ acetate nor induced a transient contraction. B, there was not a significant difference between the frequency of spontaneous contractions of the LM reduced by acetate (measurement, acetate $5 \mathrm{mM}$ ) and that after the addition of propionate (additional measurement, acetate $5 \mathrm{mM}$ + propionate $2 \mathrm{mM}$ ). Values are means $\pm \mathrm{SEM}, n=4 .{ }^{* \star} P<0.01$, significantly different from control. 


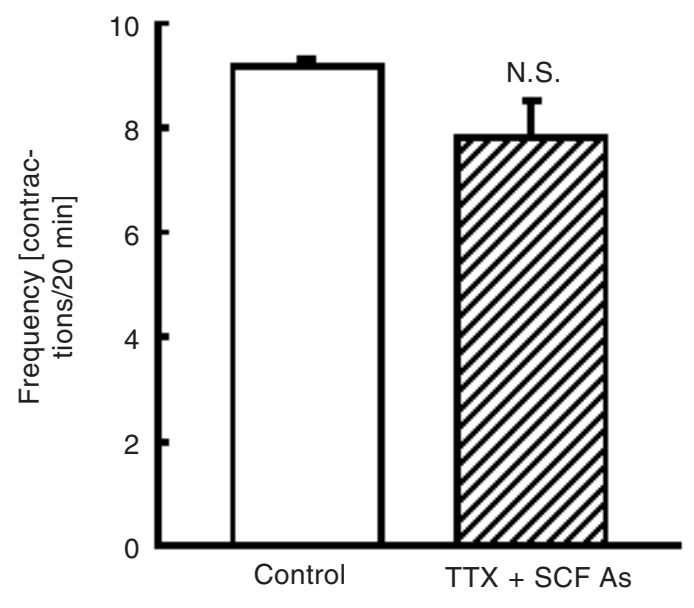

Fig. 4. Effect of TTX on SCFA-induced inhibitory response. SCFA-induced inhibitory response, which decreased the frequency of spontaneous contractions of the LM, was inhibited in the presence of $10^{-6} \mathrm{M}$ TTX. Values are means \pm SEM, $n=6$.

contractions $/ 20 \mathrm{~min}$ (measurement) $(P<0.01 \mathrm{vs}$. additional control, Fig. 5). There was a significant difference between the frequency of spontaneous contractions before the addition of L-NNA (control) and after the addition of SCFAs in the presence of L-NNA (measurement) $(P<0.05$, Fig. 5).

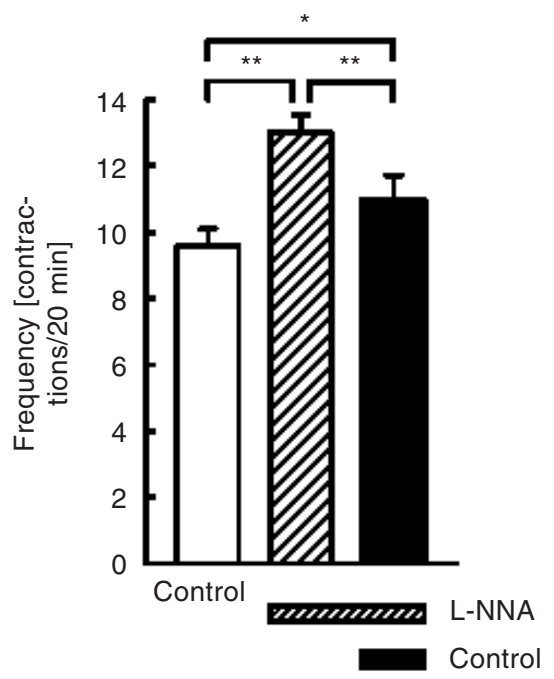

Fig. 5. Effect of L-NNA on SCFA-induced inhibitory response. In the presence of $10^{-4} \mathrm{M} \mathrm{L-NNA}$, the frequency of spontaneous contractions of the LM (control) significantly increased. SCFAs significantly decreased the frequency of spontaneous contractions in the presence of LNNA (additional control). The frequency of spontaneous contractions after the addition of SCFAs (measurement; LNNA + SCFAs) was significantly higher than the frequency of spontaneous contractions of the LM (control). The values are means \pm SEM, $n=6 .{ }^{\star} P<0.05,{ }^{\star \star} P<0.01$, significantly different from control or L-NNA (additional control).
Effects of hexamethonium and selective 5$\mathrm{HT}_{3}$ receptor antagonist on SCFA-induced inhibitory response. Recently, Nurgali et al. [22] have reported that fast synaptic input recorded from longitudinal muscle motor neurons in guinea pig distal colon is blocked by nicotinic receptor antagonist hexamethonium (C6). In the present experiments, C6 was tested to evaluate the role of nicotinic receptors in mediating SCFA-induced inhibitory response. C6 $\left(10^{-4} \mathrm{M}\right)$ itself did not affect the frequency of spontaneous contractions of the LM (Fig. 6A). In the presence of C6, SCFAs significantly decreased the frequency of spontaneous contractions of the LM from $9.2 \pm 0.3$ contractions $/ 20 \mathrm{~min}$ (control) to $7.9 \pm 0.5$ contractions/20 min (measurement) $(n=9, P<0.01$, Fig. 6D). This suggests that $\mathrm{C} 6$ alone does not affect SCFA-induced inhibitory response.

Since the participation of $5-\mathrm{HT}_{3}$ receptors in intestinal motility involving peristaltic reflex has been suggested as being especially important [23-25], the effect of 5- $\mathrm{HT}_{3}$ receptors on SCFA-induced inhibitory response was examined. Granisetron, an antagonist selective for $5-\mathrm{HT}_{3}$ receptors $\left(10^{-6} \mathrm{M}\right)$, itself did not affect the frequency of spontaneous contractions of the LM (Fig. 6B). Even in the presence of granisetron, SCFAs significantly decreased the frequency of spontaneous contractions of the LM from $9.3 \pm 0.3$ contractions $/ 20 \mathrm{~min}$ (control) to $7.8 \pm 0.6$ contractions $/ 20 \mathrm{~min}$ (measurement) $(n=6, P<0.05$, Fig. 6E). This suggests that granisetron alone does not affect SCFA-induced inhibitory response.

Granisetron, like C6, is also reported to reduce the fast excitatory synaptic potential in myenteric neurons of guinea pig distal colon [22]. Also, all neurons that contain 5-HT are immunoreactive for choline acetyltransferase (ChAT), suggesting that 5-HT and ACh could coexist [26]. Therefore the effect of the combination of C6 and granisetron on SCFA-induced inhibitory response was also investigated. The combination of $\mathrm{C} 6\left(10^{-4} \mathrm{M}\right)$ and granisetron $\left(10^{-6} \mathrm{M}\right) \mathrm{did}$ not affect the frequency of spontaneous contractions of the LM (Fig. 6C); in the presence of both C6 and granisetron, however, SCFA-induced inhibitory response was abolished ( $n=5$, Fig. $6 \mathrm{~F}$ ).

Effect of SCFAs on the frequency of spontaneous contractions of the LM in the distal colon of fed rats. To explore the possibility for the involvement of SCFAs in fecal pellets on the frequency of spontaneous contractions of the LM, we used fed rats that had fecal pellets in the distal colon. The frequency of spontaneous contractions of the LM in fed rats was $5.8 \pm 0.6$ contractions $/ 20 \mathrm{~min}(n$ 

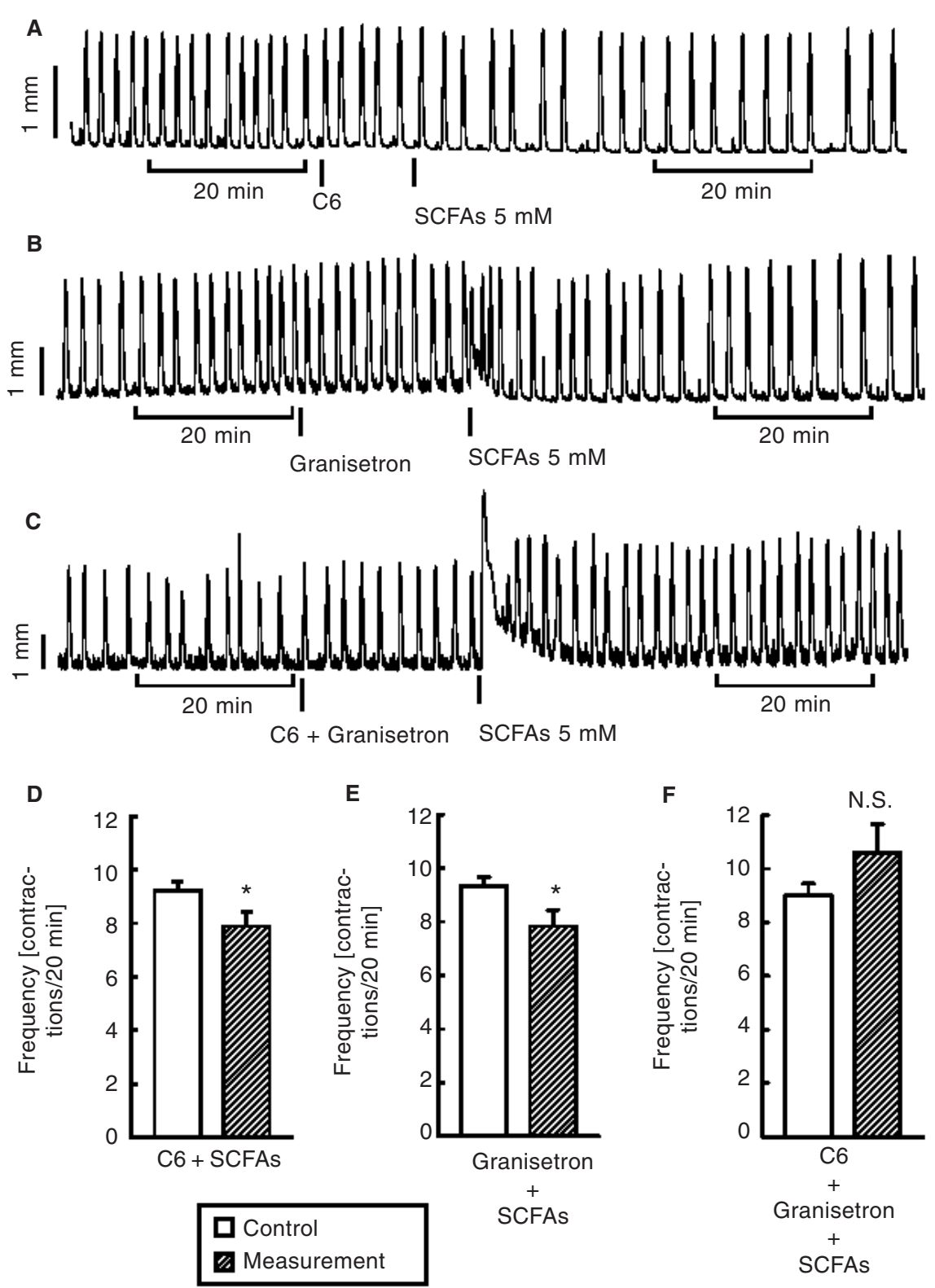

Fig. 6. Effects of $\mathbf{C 6}$ and granisetron on SCFA-induced inhibitory response. A, representative trace showing that the frequency of spontaneous contractions of the LM was not influenced by C6, but it was decreased by SCFAs in the presence of $\mathrm{C} 6\left(10^{-4} \mathrm{M}\right)$. B, representative trace showing that the frequency of spontaneous contractions of the LM was not influenced by granisetron, but it was decreased by SCFAs in the presence of granisetron (10 $\left.{ }^{-6} \mathrm{M}\right)$. C, representative trace showing that the frequency of spontaneous contractions of the LM was not influenced by C6 and granisetron and was not decreased by SCFAs in the presence of C6 $\left(10^{-4} \mathrm{M}\right)$ and granisetron $\left(10^{-6} \mathrm{M}\right)$. D, in the presence of $10^{-4}$ M C6, SCFAs significantly decreased the frequency of spontaneous contractions of the LM. The values are means \pm SEM, $n=9 .{ }^{* \star} P<0.01$, significantly different from control. E, in the presence of $10^{-6} \mathrm{M}$ granisetron, SCFAs significantly decreased the frequency of spontaneous contractions of the LM. The values are means $\pm \mathrm{SEM}, n=6$. ${ }^{\star} P<0.05$, significantly different from control. F, SCFA-induced inhibitory response was abolished in the presence of $\mathrm{C} 6\left(10^{-4} \mathrm{M}\right)$ and granisetron $\left(10^{-6} \mathrm{M}\right)$. The values are means $\pm \mathrm{SEM}, n=5$.

$=6)$. The frequency of spontaneous contractions of the LM in fed rats was a level similar to that in which the frequency of spontaneous contractions of the LM was decreased by exogenous $5 \mathrm{mM}$ SCFAs $(6.1 \pm 0.8$ contractions/20 $\min , n=8$ ); there was no statistical difference between them. The frequency of spontaneous contractions of the LM in fasted rats $(9.4 \pm 0.5$ contractions/20 $\mathrm{min}, n=7$ ) was also significantly higher compared to that in fed rats, as described in Fig. $1(n=6-7, P<0.01)$. These results raised a possibility that SCFAs contained in fecal pellets might decrease the frequency of spontaneous contractions of the LM. However, it is possible that the frequency of spontaneous contractions of the LM in fed rats may result from descending inhibition induced by the physical stimuli of fecal bulk. If the frequency of 
spontaneous contractions of the LM in fed rats is the outcome of chemical stimuli of SCFAs contained in fecal pellets, the frequency is unchanged by the further addition of exogenous SCFAs after $2 \mathrm{~h}$ equilibration. To test this idea, the LM strips from fed rats were treated with SCFAs ( $5 \mathrm{mM})$. They did not affect the frequency of spontaneous contractions of the LM in fed rats (control: $6.2 \pm 0.4$ contractions $/ 20 \mathrm{~min}$; measurement: $5.8 \pm 0.4$ contractions $/ 20 \mathrm{~min}, n=5$ ). The result indicates that the frequency of spontaneous contractions of the LM in fed rats might reflect that in a condition for the intestinal lumen to be satisfactorily exposed by SCFAs.

\section{DISCUSSION}

This study has examined the effects of SCFAs on the frequency of spontaneous contractions of the LM in rat distal colon. It is thought that the spontaneous contractions of the LM as well as that of the CM are peristaltic in nature. In the present study, we have shown that exogenous SCFAs ( $>5 \mathrm{mM}$ ) decrease the frequency of spontaneous contractions of LM in rat distal colon. These results suggest that the frequency of spontaneous contractions of the LM in rat distal colon is decreased by SCFAs in the intestinal lumen; the frequency of spontaneous contractions may be always inhibited by SCFAs produced by bacterial fermentation in the colon. Therefore it appears likely that SCFAs, chemical substances in the intestinal lumen, are detected by the colon and influence colonic motility in addition to the physical stimuli of fecal bulk in rat distal colon.

Acetate as a substantial stimulus having a significant effect in rat distal colon. Among individual SCFAs, only acetate decreased the frequency of spontaneous contractions of the LM (Fig. 2 ). Thus it is suggested that acetate appears to be a substantial stimulus of SCFA-induced inhibitory response in rat distal colon. In rat terminal ileum, acetic acid, but not acetate, induces the contraction of LM in a concentration-dependent fashion [27]. In the colon, however, the significant effects of acetate or acetic acid are as yet unknown. It has been reported that acetate neither induces a transient contraction in the LM in rat colon [7] nor modifies colonic motor patterns in rats [8]. In the present study, acetate induced a decrease in the frequency of spontaneous contractions in rat distal colon (Fig. 2, A and D).

In contrast to acetate, in the present study propionate increased the frequency of spontaneous contractions of the LM. Although SCFAs contain propionate in addition to acetate and butyrate, they induced a decrease in the frequency of spontaneous contractions of the LM (Fig. 1). From these results, it can be surmised that the effect of propionate increasing the frequency of spontaneous contractions of the LM may be masked in the presence of acetate. It has been reported that propionate-induced transient contraction is abolished by pretreatment with acetate [7]. In the presence of acetate in the present study, propionate did not cause a further increase or decrease in the frequency of spontaneous contractions of the LM in the colon (Fig. 3). These data support the idea that acetate prevents the effect of propionate increasing the frequency of spontaneous contractions of the LM in rat distal colon. Therefore it appears that SCFAs under physiological conditions usually induce a decrease in the frequency of spontaneous contractions of the LM in rat distal colon.

In some cases, SCFAs evoked a transient contraction immediately after their addition. It has been reported that propionate and butyrate, but not acetate, cause a transient contraction of LM strips of rat colon and that this transient contraction is abolished by pretreatment with acetate [7]. Therefore the transient contraction induced by SCFAs in the present study is considered to be caused by propionate and butyrate. In the cases in which SCFAs did not evoke a transient contraction, this contraction might have been masked by the presence of acetate, similar to the result in which pretreatment with acetate abolished the propionate-induced increase in the frequency of spontaneous contractions of the LM.

Involvement of enteric neurons on SCFAinduced inhibitory response. SCFA-induced inhibitory response was abolished by TTX (Fig. 4). Thus it is suggested that this response is mediated via the ENS. It has been reported that a constitutive release of NO from enteric neurons suppresses the frequency of spontaneous contractions in rat colon [12]. In the present study, L-NNA itself significantly increased the frequency of spontaneous contractions in rat distal colon (Fig. 5), suggesting the existence of tonic inhibitory inputs to the LM, which decrease the frequency of spontaneous contractions. This observation is in agreement with the previous finding [12]. However, in the present study we could not identify an inhibitory transmitter relating to SCFAinduced inhibitory response because the NOS inhibitor did not abolish SCFA-induced inhibitory response. Cherbut et al. [8] have reported that 500 $\mathrm{mM}$ of SCFAs, propionate, and butyrate decrease the ratio of orally to aborally propagated activity to accelerate colonic transit through a neuroendocrine mechanism involving PYY, but $100 \mathrm{mM}$ of SCFAs and 
acetate itself have no effect. The present study demonstrated that the concentration of SCFAs $(5 \mathrm{mM})$ needed for SCFA-induced inhibitory response was much smaller than the $500 \mathrm{mM}$ concentration reported by Cherbut et al. [8], and that among SCFAs, acetate was a substantial stimulus in SCFA-induced inhibitory response. Therefore it seems that PYY is probably not involved in the mechanism mediating SCFAinduced inhibitory response.

Since SCFA-induced inhibitory response seems to be linked with the ENS, in the present study we have further analyzed the involvement of the ENS using nicotinic and 5-HT receptor antagonists on SCFAinduced inhibitory response. In the present study, neither C6 nor granisetron affected SCFA-induced inhibitory response (Fig. 6, A, B, D, and E). However, the combination of $\mathrm{C} 6$ and granisetron abolished SCFA-induced inhibitory response (Fig. 6, C and F). Thus nicotinic and $5-\mathrm{HT}_{3}$ receptors are quite likely to be involved in the response. This is supported by the following observations: (1) All enteric neurons in the myenteric plexus, except NOS-immunoreactive inhibitory muscle motoneurons, contain ChAT [26]; (2) 5-HT is released from EC cells in response to SCFAs and stimulates $5-\mathrm{HT}_{3}$ receptors on the vagal afferent fibers [9]; and (3) $5-\mathrm{HT}_{3}$ and 5- $\mathrm{HT}_{4}$ receptors mediate the contraction and relaxation of the LM of guinea pig distal colon [23]. In the present study, we could not identify the location of these receptors in the ENS. Therefore further studies will be necessary to clarify the locations of the receptors and the sensory mechanism of SCFAs.

Functional significance of SCFA-induced inhibitory response of the $L M$ in rat distal colon. In the present study, we showed that exogenous SCFAs decreased the frequency of spontaneous contractions of the LM via the enteric nerves involving nicotinic and $5-\mathrm{HT}_{3}$ receptors. These observations support our hypothesis that the frequency of spontaneous contractions of the LM in rat distal colon may be always inhibited by SCFAs in intestinal lumen. Thus it is suggested that when the whole of rat distal colon is exposed to adequate SCFAs produced in the colon under physiological conditions, SCFAs reduce the frequency of spontaneous contractions of the LM and may facilitate the movement of fecal pellets. This is because it has been reported that the movement of artificial fecal pellets through guinea pig distal colon is smoothest in preparations where the frequency of rhythmic peristaltic waves, namely, CMMCs, is low [28]. Therefore we propose that SCFAs produced by colonic fermentation under physiological conditions ( $\sim 100 \mathrm{mM})$ may mainly play an important role in regulating the frequency of spontaneous contractions of the LM to facilitate colonic motility, including the peristaltic reflex in rat distal colon.

Roles for SCFAs contained in fecal pellets in the frequency of spontaneous contractions of the LM. The present study showed that the frequency of spontaneous contractions of the LM in the distal colon treated with exogenous $5 \mathrm{~mm}$ SCFAs was similar to that in fed rats. Furthermore, we showed that an exogenous addition of $>5 \mathrm{mM}$ SCFAs to the basting solution did not decrease the frequency of spontaneous contractions of the LM in fed rats. From the results, we thought that the decrease in the frequency of spontaneous contractions of the LM by exogenous SCFAs might be similar to the result because of the exposure of colonic mucosae to SCFAs contained in fecal pellets for the following reasons: (1) The influence of intraluminal fluid distension used as physical stimuli is eliminated within 10-15 min after the infusion pump is stopped in the guinea pig distal colon [10]; (2) An artificial fecal pellet can be inserted at $5 \mathrm{~min}$ intervals in isolated segments of guinea pig colon [29]. These reports suggest that the influence of physical stimuli provided by fecal bulk is transient and that after the fecal pellets are removed from rat distal colon, their influence is easily eliminated during $2 \mathrm{~h}$ equilibration. Therefore it will be considered that in the present study, physical stimuli provided by fecal bulk is not a primary inhibitory stimulus. Because SCFAs are thought to migrate with fecal pellets in the middle and distal colon, it is reasonable to speculate that the frequency of spontaneous contractions of the LM in rat distal colon might always be inhibited by SCFAs contained in fecal pellets. Furthermore, it is very likely that rat distal colon has a character in which the frequency of spontaneous contractions of the LM might be altered by the presence of SCFAs contained in fecal pellets, because the frequency of spontaneous contractions of the LM in fasted rats was significantly higher than that in fed rats; the frequency of spontaneous contractions of the LM increases in the absence of SCFAs and decreases in the presence of SCFAs. However, to understand the more precise roles for SCFAs contained in fecal pellets in the frequency of spontaneous contractions of the LM, further studies will be needed.

Conclusion. In conclusion, the present study suggests that (1) the frequency of spontaneous contractions of the LM in rat distal colon is decreased by SCFAs; (2) exogenous SCFA-induced inhibitory response is mediated through the ENS, which involves 
nicotinic and 5- $\mathrm{HT}_{3}$ receptors; and (3) this phenomenon might be mediated by SCFAs present in fecal pellets.

\section{REFERENCES}

1. Cummings JH, Pomare EW, Branch W, Naylor CPE, and Macfarlane GT: Short chain fatty acids in human large intestine, portal, hepatic and venous blood. Gut 28: 1221-1227, 1987

2. Kamath PS, Hoepfine MT, and Phillips SF: Short-chain fatty acids stimulate motility of the canine ileum. Am J Physiol 253: G427-G433, 1987

3. Richardson A, Delbridge AT, Brown NJ, Rumesey RDE, and Read NW: Short chain fatty acids in the terminal ileum accelerate stomach to caecum transit in the rat. Gut 32: 266-269, 1991

4. Ropert A, Cherubut C, Roze C, Le Quellec A, Holst JJ, Fu-cheng X, Bruley des Varannes S, and Galmiche JP: Colonic fermentation and proximal gastric tone in human. Gastroenterology 111: 289-296, 1996

5. Cuche $\mathrm{G}$, and Malbert $\mathrm{CH}$ : Short-chain fatty acids present in the ileum inhibit fasting gastrointestinal motility in conscious pigs. Neurogastroenterol Motil 11: 219-225, 1999

6. Cuche G, Cuber JC, and Malbert CH: Ileal shortchain fatty acids inhibit gastric motility by a humoral pathway. Am J Physiol 279: G925-G930, 2000

7. Yajima T: Contractive effect of short-chain fatty acids on the isolated colon of the rat. J Physiol (London) 368: 667-678, 1985

8. Cherbut C, Ferrier L, Roze C, Anini Y, Blottiere H, Lecannu G, and Galmiche JP: Short-chain fatty acids modify colonic motility through nerves and polypeptide $Y Y$ release in the rat. Am J Physiol 275: G1415-G1422, 1998

9. Fukumoto S, Tatewaki M, Yamada T, Fujimiya M, Mantyh C, Voss M, Eubanks S, Harris M, Pappas TN, and Takahashi T: Short-chain fatty acids stimulate colonic transit via intraluminal $5-\mathrm{HT}$ release in rats. Am J Physiol 284: R1269-R1276, 2003

10. Smith TK, and Robertson WJ: Synchronous movements of the longitudinal and circular muscle during peristalsis in the isolated guinea-pig distal colon. J Physiol (London) 506: 563-577, 1998

11. Macfarlane GT, and Gibson GR: Microbiological aspects of the production of short-chain fatty acids in the large bowel. In: Physiological and Clinical Aspects of Short-Chain Fatty Acids, ed. Cummings $\mathrm{JH}$, Rombeau JL, and T Sakata T, Cambridge University Press, Cambridge, pp 87-105, 1995

12. Gonzalez A, and Sarna SK: Neural regulation of in vitro giant contractions in the rat colon. Am J Physiol 281: G275-G282, 2001

13. Gonzalez A, and Sarna SK: Different types of contractions in rat colon and their modulation by oxidative stress. Am J Physiol 280: G546-G554, 2001

14. Pluja L, Alberti E, Fernandez E, Mikkelsen HB, Thuneberg L, and Jimenez M: Evidence supporting presence of two pacemakers in rat colon. Am J
Physiol 281: G255-G266, 2001

15. Powell AK, and Bywater RAR: Murine intestinal migrating motor complexes: longitudinal components. Neurogastroenterol Motil 15: 245-256, 2003

16. Powell AK, and Bywater RAR: Endogenous nitric oxide release modulates the direction and frequency of colonic migrating motor complexes in the isolated mouse colon. Neurogastroenterol Motil 13: 221-228, 2001

17. Sethi AK, and Sarna SK: Contractile mechanisms of colonic propulsion. Am J Physiol 268: G530-G538, 1995

18. Matsufuji H, Yokoyama J, Hirabayashi T, Watanabe S, and Sakurai S: Cooperative roles of colon and anorectum during spontaneous defecation in conscious dogs. Dig Dis Sci 43: 2042-2047, 1998

19. Malcolm A, and Camilleri M: Coloanal motor coordination in association with high-amplitude colonic contractions after pharmacological stimulation. Am J Gastroenterol 95: 715-719, 2000

20. Sakata T, and von Engelhardt W: Influence of short chain fatty acids and osmolality on the mucin release in the rat colon. Cell Tissue Res 219: 371-377, 1981

21. von Engelhardt W: Absorption of short-chain fatty acids from the large intestine. In: Physiological and Clinical Aspects of Short-Chain Fatty Acids, ed. Cummings JH, Rombeau JL, and Sakata T, Cambridge University Press, Cambridge, $p p$ 149-170, 1995

22. Nurgali K, Furness JB, and Stebbing MJ: Analysis of purinergic and cholinergic fast synaptic transmission to identified myenteric neurons. Neuroscience 116: 335-347, 2003

23. Woollard DJ, Bornstein JC, and Furness JB: Characterization of 5-HT receptors mediating contraction and relaxation of the longitudinal muscle of guinea-pig distal colon in vitro. Naunyn Schmiedebergs Arch Pharmacol 349: 455-462, 1994

24. Briejer MR and Schuurkes JAJ: 5-HT3 and 5-HT4 receptors and cholinergic and tachkininergic neurotransmission in the guinea-pig proximal colon. Eur J Pharmacol 308: 173-180, 1996

25. Kadowaki M, Wade P, and Gershon MD: Participation of 5-HT3, 5-HT4 and nicotinic receptors in the peristaltic reflex of guinea pig distal colon. Am J Physiol 271: G849-G857, 1996

26. Funess JB: Types of neurons in the enteric nervous system. J Auton Nerv Syst 81: 87-96, 2000

27. Cherbut C, Aube AC, Blottiere HM, Pacaud P, Scarpignato C, and Galmiche JP: In vitro contractile effects of short chain fatty acids in the rat terminal ileum. Gut 38: 53-58, 1996

28. Smith TK, Oliver GR, Hennig GW, O'Shea DM, Berghe $\mathrm{PV}$, Kang SH, and Spencer NJ: A smooth muscle tone-dependent stretch-activated migrating motor pattern in isolated guinea-pig distal colon. J Physiol (London) 551: 955-969, 2003

29. Foxx-Orenstein AE, and Grider JR: Regulation of colonic propulusion by enteric excitatory and inhibitory neurotransmitters. Am J Physiol 271: G433-G437, 1996 